\title{
Basic Theories and Applications of Digital Image Processing
}

\author{
Jian Guo ${ }^{1, \text { a }}$ \\ ${ }^{1}$ School of Engineering, University of Bridgeport, Connecticut 06604, US; \\ jianguo@my.bridgeport.edu
}

Keywords: Digital image processing; applications; basic theory.

\begin{abstract}
Nowadays, with the rapid development of science and technology, computer takes place over human production and life. Digital image processing technology which utilizes computer to deal with digital images has emerged and has been widely used in various fields, such as remote sensing aerospace, biomedical engineering, communication engineering, industry and engineering, military, etc. Image is a relatively comprehensive and convenient way for humans to receive, express, and transmit information. Digital image processing refers to the use of digital computers to process images. Based on the basic theories of digital image processing, we summarize the application of digital image processing and expound its development and trend in the future.
\end{abstract}

\section{Introduction}

Vision is a way of human perception of the world, it is an important means for people to obtain and express information from nature. In the information that human obtains from nature, auditory information accounts for $20 \%$, visual information $60 \%$, and other information about $20 \%$. Image is specific information that is conveyed visually to the human being, and it is a similarity description of the objectively existing things of nature. Image contains substantial content and information, such as color, texture, shape, edge, etc. These provide bases for us to better understand and use images, and we can use them to meet some of our needs. Therefore, how to deal with a piece of images to meet the various needs of humankind has become the focus of people's research, thus image processing technology came into being.

The so-called digital image processing refers to using digital computers to treat and process images. The purpose is to restore the original features of image, to improve the visual effects of people, to highlight the characteristics of some objects in image and extract the characteristic parameters of the targets [1]. In terms of discipline, the main contents of research are image digitization, image transformation, image enhancement, image restoration, image compression(coding), image segmentation, image description and image classification (recognition). Herein, image digitization refers to discretizing an analog image to a digital image. Image transformation is a technique that simplifies image processing and improves the performance of image processing. Image enhancement refers to highlighting or sharpening certain features (such as edges, contours, contrast, etc.) of an image, in order to display, observe, or further analysis and process the image. Image restoration is the restoration of the degraded, blurred image. Based on the characteristics (such as gray, color, texture, edge, etc.) of image, image segmentation means that divides some meaningful features and extracts them for further processing, such as image recognition and analysis. Image description is to use a simpler and definite numerical, symbolic or graphical representation of the attributes of the segmented image regions and the relationship between them, and it is a necessary prerequisite for image recognition and understanding. Image classification (recognition) belongs to the category of pattern recognition and the main contents are as follows: 1.image preprocessing (enhancement, restoration, compression); 2.image segmentation and feature extraction; 3.judgment and classification.

The earliest digital image processing was accompanied by the development of electronic computers in the twentieth century. At the beginning, image processing was based on the criterion of human visual perception to improve the effect of the image, mainly to deal with it as a high quality 
image that in line with the human visual requirements. In the 1980s and 1990s, digital image processing developed rapidly. In 1988, Mallat used wavelet analysis to effectively decompose and reconstruct the image. Wavelet analysis was considered as an important breakthrough in the field of signal and image analysis. After that, digital image processing technology developed explosively [2]. Since the 21st century, with the rapid development of computer technology and gradual improvement of relevant theories, digital image processing technology tended to be real-time, intelligent, network and three-dimensional, and widely recognized and used in a variety of fields. Today, image processing technology is successfully used in industrial, health care, aerospace, military and other fields, and plays an increasingly important role, and achieves significant social and economic benefit.

\section{The Basic Theory of Digital Image Processing}

From the end of the 20th century to the present and even for some time in the future, digital image processing is mainly focused on searching for new theories and algorithms, mainly reflected in the following ways.

\subsection{Wavelet Theory.}

Wavelet is called the "microscope" of mathematics, compared to Fourier transform, it has great advantages. In the 1980s, France' Mallat proposed Mallat algorithm and Meyer of the United States established a multi-resolution analysis, the application of wavelet theory achieved a real breakthrough. After ten years of development, although its application has get certain development, but there are still several problems to be resolved.

1). The construction of wavelet basis. At present, a better wavelet is the Daubechies wavelet created by Daubechies of the Belgian [3]. Finding an appropriate wavelet base has always been a very tricky problem. People have not yet found an ideal method, it is still an urgent need to solve the problem of wavelet theory. It is generally believed that wavelet theory is still in its early stage.

2). The application of multidimensional wavelet. At present, only the two-dimensional wavelet is used, and it is the one-dimensional wavelet in most cases, multidimensional wavelet still remains in the theoretical discussion stage. In fact, the application of multidimensional wavelet is very extensive, such as remote sensing image is affected by the location, wavelength and time, ie pixels $=f(x, y, z, \lambda, t)$. If we use a five-dimensional wavelet to process remote sensing digital images, the result is better than using the two-dimensional wavelet.

\subsection{Fractal Theory.}

Fractal geometry is proposed by B.B.Mandelbort of the British in the 1970s [4]. The term "fractal" is also proposed by B. B. Mandelbort, which contains the meaning of "irregular" and "broken". Fractal theory is one of the main branches of nonlinear science. It is widely used in various disciplines of natural sciences, even in economic and social activities.

All shapes in nature and all the patterns that people consider can be divided into two categories: 1) those with characteristic scales, such as human height, sphere radius, etc. 2) those without feature scales, that is, many scales (from small to large) must be considered, such as cumulonimbus, frost and so on. The so-called "scale-free" geometry is actually fractal. They have a common characteristic, ie self-similarity. In the image, there are a lot of fractal geometry, such as rivers, coastlines, etc., so digital image processing uses a lot of fractal theories. The main difficulty of fractal theory is self-similarity and dimension. Strictly speaking, each geometry is self-similar, but how to find self-similarity is a difficult point. At present, the fractal theory for image compression is more mature.

\subsection{Artificial Neural Theory.}

Artificial Neural Network (ANN) is a designed machine to imitate the way the human brain works. It can be implemented with electronic or optoelectronic components and can also be simulated by software on a conventional computer [5]. In other words, ANN is a parallel distributed processor with a large number of connections, it has the ability to acquire knowledge and solve problems by learning. Since the early 1980s, the study of the neural network began to develop rapidly, it has achieved fruitful results in terms of its computational power, the approximation ability of any continuous 
mapping, the learning theory and the stability analysis of the dynamic network. Especially the application has been promptly extended too many important areas.

In digital image processing, ANN has been successfully applied on printing and handwriting character recognition, speech recognition, signature recognition, fingerprint, face recognition, RNA and DNA sequence analysis, cancer cell identification, target detection and recognition, electrocardiogram and EEG classification, image restoration and recovery, and so on.

Although the ANN has made some achievements in digital image processing, but it is still in the initial stage. Firstly, the people understanding on the brain neural network structure and operation mechanism, and even the working principle of a single nerve cell is very shallow. Although a certain primary intelligent artificial neural network has been constructed, which is based on the biological nervous system of distributed storage, parallel processing, and adaptive learning these phenomena. However, this ANN is only a rough and simple simulation of the brain, whether on the scale or functionally is far from brain. Secondly, the number of hidden layers in the neural network, the number of neurons, the methods of learning and other important issues have not been completely resolved, so its research and application still remain in the initial stage.

\section{The Applications and Trends of Digital Image Processing}

\subsection{The Applications of Digital Image Processing.}

Image appears in all areas of human production and life, so the application of digital image processing also involves all aspects of human production and life, mainly in the following fields:

1) Biomedical field: Digital image processing has been maturely used in the field, and showed good application value and prospects. Such as the beginning CT technology, later technologies that applied to the detection and counter of certain cells, cancer cell detection, identification, and chromosome analysis, etc. These are better to help the doctor check the patient. In addition, digital image processing is also widely used in expert systems, DNA analysis, X-ray images, enhancement of the ultrasound image.

2) Aerospace field: The successful mapping of digital image processing technology on the moon opened its development in the field. At present, digital image processing technology is widely used in aerial remote sensing and satellite remote sensing, in addition to satellite image processing. Through the processing and analysis of satellite images, extracting a large number of useful information rapidly, and eventually used for resource surveys, resource exploration, disaster detection, terrain, map census and mapping, weather forecast.

3) Military and public security fields: In the military field, digital image processing technology can be used for radar target detection, cruise missile terrain identification, remote control aircraft guidance, simulation training system. As in the public security, digital image processing technology can be used for the extraction of physical evidence, the synthesis of criminal face, fingerprint recognition, license plate recognition, and traffic monitoring, accident analysis, etc. Digital image processing can provide reliable evidence for police officers to help them solve the case quickly.

4) Communication field: Nowadays, in the image of the remote communication, digital image processing shows its important role. As one of the main contents of the current communication, image becomes extremely difficult because of its huge amount of data. In order to make such a large amount of data to be transmitted in real time, it is necessary to use compression technology to compress the information. Through entropy coding, network coding and wavelet coding method in digital image processing can realize the efficient transmission of image information.

5) Cultural and artistic fields: In the culture field, digital image processing can be used for the protection \&\& repairing of ancient damaged murals, and the copy\&\&repairing of photographs in valuable cultural relics, etc. In the field of art, computer art has become a new art, digital image processing is used in clothing, hairstyle, housing and other designs. In addition, digital image processing is also used for animation production, athlete action analysis and rating, and so on.

\subsection{The Trend of Digital Image Processing.}

Today, digital image processing technology has made great achievements and obtained a good application in many areas. However, with the development of human society, the technology in 
practical application also faces many new challenges. In the future, the technology will be further improved in the following aspects: (1) Improve the accuracy of image processing, and put forward a set of image processing evaluation criteria to minimize the subjective evaluation. (2) Optimize algorithms, update theories and establish optimal mathematical models to improve the image processing speed; in the face of large amounts of data, while ensuring accuracy, reduce data size and data operations, achieving further real-time image processing. (3)Further improve the theoretical system, develop a centralized, intelligent, rational operation platform, and so that digital image processing technology can be extend to other disciplines to achieve interdisciplinary development.

\section{Conclusions}

A digital image is an image represented by a matrix and centered on a pixel. With the rapid development of science and technology, computer takes place over human production and life. Digital image processing technology utilizes computer to deal with digital images to meet human needs has emerged and has been widely used in various fields. Based on the basic theory and research of digital image processing, we summarize the application of digital image processing and expound its trend in the future.

\section{References}

[1] Jain, A. K. (2011). Fundamentals of digital image processing.Fundamentals of digital image processing : Wiley.

[2] Mallat, S. (1998). A Wavelet Tour of Signal Processing, Third Edition: The Sparse Way. Academic press.

[3] Lina, J. M., \& Mayrand, M. (1995). Complex daubechies wavelets.Applied \& Computational Harmonic Analysis, 2(3), 219-229.

[4] Blumen, A., \& Mandelbort, B. B. (1989). Fractals, geometry: what is it, and what does it do?: discussion. Proceedings of the Royal Society A Mathematical Physical \& Engineering Sciences, 423(1864), 16.

[5] Hassoun, M. H. (1996). Fundamentals of artificial neural networks.Proceedings of the IEEE, 84(6), 906. 